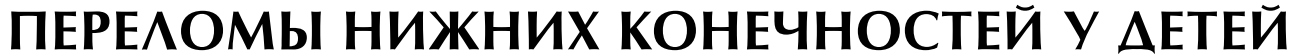 С ПОСАЕАСТВИЯМИ СПИННОМОЗГОВЫХ ГРЫЖ
}

\author{
(C) С.В. Иванов ${ }^{1}$, В.М. Кенис ${ }^{1}$, Т.Н. Прокопенко ${ }^{1}$, А.С. Федосеева ${ }^{2}$, М.А. Угурчиева \\ ${ }^{1}$ ФГБУ «НИДОИ им. Г.И. Турнера» Минздрава России, Санкт-Петербург; \\ ${ }^{2}$ СПбГБУЗ «Детская городская больница Святой Ольги», Санкт-Петербург; \\ ${ }^{3}$ ФГБОУ ВО «Первый Санкт-Петербургский государственный медицинский университет \\ имени академика И.П. Павлова» Минздрава России, Санкт-Петербург
}

\begin{abstract}
Введение. Спинномозговая грыжа - это тяжелый порок развития позвоночника и спинного мозга, сопровождающийся разнообразными ортопедическими нарушениями со стороны позвоночника и нижних конечностей, в том числе переломами длинных трубчатых костей. При спинномозговых грыжах остеопороз имеет ключевое значение в патогенезе двигательных нарушений.

Цель исследования - определить закономерности возникновения и клинико-рентгенологические особенности переломов длинных трубчатых костей нижних конечностей у детей с последствиями спинномозговой грыжи.

Материалы и методы. В ФГБУ «НИДОИ им. Г.И. Турнера» Минздрава России в период с 2006 по 2017 г. проведено обследование и лечение 544 пациентов с последствиями спинномозговых грыж. Применяли клинико-неврологический и рентгенологический методы исследования. Определяли нейросегментарный уровень поражения спинного мозга, используя классификацию Sharrard, а также двигательный уровень по методике, предложенной Мельбурнским медицинским университетом.

Результаты. Клиническая картина перелома длинной трубчатой кости у ребенка со спинномозговой грыжей имеет ряд особенностей. Патологической подвижности в месте перелома не было в 56 \% случаев, отек отсутствовал у 88 \% детей, а боль в области перелома мы наблюдали только в 19 \% случаях. Среди рентгенологических особенностей атипичного перелома длинных трубчатых костей у детей с последствиями спинномозговых грыж выделялись: отсутствие «линии» перелома, наличие гипертрофированной периостальной реакции, а также участки склероза в месте перелома.

Заключение. Частота и локализация переломов нижних конечностей у детей с последствием спинномозговой грыжи определяются нейросегментарным уровнем. Клиническая картина перелома зачастую отличается от обычных переломов отсутствием болевого синдрома, отека в области перелома, смещения костных отломков, что необходимо учитывать при диагностике. Особенности клинико-рентгенологической картины связаны с наличием при данной патологии остеопороза вследствие снижения уровня двигательной активности пациентов.
\end{abstract}

Ключевые слова: спинномозговая грыжа; перелом бедренной и большеберцовой кости; остеопороз у детей.

\section{FRACTURES OF LOWER LIMBS IN CHILDREN WITH SPINA BIFIDA}

(C) S.V. Ivanov ${ }^{1}$, V.M. Kenis ${ }^{1}$, T.N. Prokopenko ${ }^{1}$, A.S. Fedoseeva ${ }^{2}$, M.A. Ugurchieva ${ }^{3}$

${ }^{1}$ The Turner Scientific Research Institute for Children's Orthopedics, Saint Petersburg, Russia;

${ }^{2}$ Children's City Hospital of St. Olga, Saint Petersburg, Russia

${ }^{3}$ Pavlov First Saint Petersburg State Medical University, Saint Petersburg, Russia

For citation: Pediatric Traumatology, Orthopaedics and Reconstructive Surgery. 2018;6(3):25-31

Received: 05.06.2018 Accepted: 04.09.2018

Background. Spina bifida is a serious defect in the development of the spine and spinal cord. It is accompanied by several orthopedic disorders of the spine and lower limbs, including fractures of long tubular bones. In spina bifida, osteoporosis plays an important role in the pathogenesis of motor disorders. 
Aim. The objective was to determine the patterns of occurrence and the clinical and radiological features of fractures of the long tubular bones of the lower limbs in children with sequelae of spina bifida.

Materials and methods. From 2006 to 2017, 544 patients with spina bifida were examined and treated at the Turner Research Institute for Children's Orthopedics. Clinical-neurological and radiographic methods were used. The neurosegmental level of spinal cord involvement was determined using the Sharrard classification, and the motor level was assessed according to the method proposed by Melbourne Medical University.

Results. The clinical picture of a fracture of a long tubular bone in a child with spina bifida has many characteristics. There was no abnormal mobility in the fracture site in $56 \%$ of cases, edema was absent in $88 \%$ of children, and pain in the fracture region was observed in only $19 \%$ of cases. The radiographic features of the atypical fracture of long tubular bones in children with sequelae of spina bifida included lack of a fracture line, presence of a hypertrophic periosteal reaction, and sclerosis areas at the fracture site.

Conclusion. The frequency and localization of fractures of the lower limbs in children with sequelae of spina bifida are determined according to the neurosegmental level. The clinical picture of fracture often differs from usual fractures by the absence of pain syndrome, edema in the fracture region, and displacement of bone fragments, which must be considered for diagnosis. The peculiarities of the clinical and radiological picture are associated with the presence of osteoporosis in this pathology due to a decrease in the motor activity level of the patients.

Keywords: spina bifida; fracture of the femur and the tibia; osteoporosis in children.

\section{Введение}

Спинномозговая грыжа - порок развития позвоночника, представляющий собой неполное закрытие нервной трубки в не полностью сформированном спинномозговом канале, сопровождающийся разнообразными ортопедическими нарушениями со стороны позвоночника и нижних конечностей. В процессе лечения деформаций нижних конечностей, вызванных в основном мышечным дисбалансом из-за нейротрофических нарушений, статико-динамических изменений, нередко возникают переломы длинных трубчатых костей [1-3]. За последние десятилетия интерес к изучению остеопороза у детей значительно повысился [4]. Высокий риск переломов костей у пациентов с нейромышечными заболеваниями широко обсуждается в литературе. С точки зрения патогенеза выделяют два варианта остеопороза: первичный и вторичный. При первичном остеопорозе отсутствует верифицированная причина. Вторичный остеопороз развивается как следствие основного заболевания. Важнейшие факторы при моделировании костной ткани - это мышечная активность и осевые нагрузки. Оба эти фактора необходимы для нормального формирования костной архитектоники и сохранения костной массы. При спинномозговых грыжах остеопороз имеет ключевое значение в патогенезе двигательных нарушений [5].

Факторами риска остеопороза у детей с последствиями спинномозговой грыжи являются снижение двигательной активности, снижение осевой нагрузки, иммобилизация после оперативного лечения, нарушения питания, низкие темпы роста. Иммобилизация пациента или конечности приводит к повышению остеокластической активности и увеличению продукции цитокининов $[6,7]$. В то же время у детей с последствиями спинномозговых грыж могут иметь место переломы, трактующиеся как патологические. Как правило, эти переломы возникают в результате форсированных движений при проведении восстановительного лечения. Рентгенологическая картина перелома у данной группы пациентов отличается рядом характерных особенностей, наиболее важной из них является формирование избыточной костной мозоли, наличие которой у ребенка с последствиями спинномозговой грыжи вызовет необходимость проведения дифференциальной диагностики с опухолью, остеомиелитом и другими заболеваниями костей $[8,9]$.

Цель исследования - определить закономерности возникновения и клинико-рентгенологические особенности переломов длинных трубчатых костей нижних конечностей у детей с последствиями спинномозговой грыжи.

\section{Материалы и методы}

Объектом исследования стали 544 ребенка в возрасте от периода новорожденности до 17 лет, находившиеся на обследовании и лечении в отделении нейроортопедии и системных заболеваний НИДОИ им. Г.И. Турнера в период с 2006 по 2017 г. по поводу ортопедических деформаций нижних конечностей, вызванных последствиями спинномозговой грыжи. Применялись анамнестический, клинический и рентгенологический методы исследования.

При сборе анамнеза у всех пациентов уточняли сведения о переломах длинных трубчатых костей нижних конечностей. При клиническом обследо- 
К^ассификация Авигательных нарушений по нейросегментарному уровню по Sharrard

\begin{tabular}{|c|l|c|}
\hline $\begin{array}{c}\text { Уровень } \\
\text { двигательных } \\
\text { нарушений }\end{array}$ & \multicolumn{1}{|c|}{ Мышечная сила } & $\begin{array}{c}\text { Нейросегментарный } \\
\text { уровень }\end{array}$ \\
\hline 1 & Сила подошвенных сгибателей стопы (4-5 баллов) & $\mathrm{S}_{2}$ \\
\hline 2 & $\begin{array}{l}\text { Сила подошвенных сгибателей стопы (менее 3 баллов) } \\
\text { Сила сгибателей голени (3 балла) } \\
\text { Сила разгибателей и/или отводящих мышц бедра (2-3 балла) }\end{array}$ & $\mathrm{S}_{1}-\mathrm{L}_{5}$ \\
\hline 3 & $\begin{array}{l}\text { Сила сгибателей бедра (4-5 баллов) } \\
\text { Сила сгибателей голени (менее 3 баллов) } \\
\text { Сила разгибателей и отводящих мышц бедра (1-2 балла) }\end{array}$ & $\mathrm{L}_{4}-\mathrm{L}_{3}$ \\
\hline 4 & $\begin{array}{l}\text { Отсутствие активного разгибания в коленном суставе } \\
\text { Сила сгибателей бедра (приводящих мышц) (менее 2 баллов) } \\
\text { Поднятие таза (3-4 балла) }\end{array}$ & $\mathrm{L}_{2}-\mathrm{L}_{1}$ \\
\hline 5 & $\begin{array}{l}\text { Отсутствие мышечной активности нижних конечностей. } \\
\text { Отсутсвие способности приподнять таз над опорной поверхностью } \\
\text { в горизонтальном положении }\end{array}$ & Грудной \\
\hline
\end{tabular}

вании определяли нейросегментарный уровень последствий спинномозговой грыжи согласно классификации, предложенной Sharrard [10]. Она основана на оценке силы основных мышечных групп нижних конечностей (в баллах), иннервирующихся соответствующим нейросегментом (табл. 1).

У всех пациентов также определялся двигательный уровень по методике, предложенной Мельбурнским медицинским университетом для детей с последствиями спинномозговых грыж [11], которая включает пять уровней двигательной активности: уровень 5 - способность ходить не хуже сверстников; уровень 4 - ходьба с использованием ортезов голеностопного сустава без дополнительных средств опоры, использование коляски только на длинные дистанции; уровень 3 - ходьба в пределах дома с помощью ортезов и дополнительных средств опоры, использование коляски как вне, так и внутри дома; уровень 2 - ходьба во время лечения, в школе и ограниченное время дома, использование коляски при передвижении и уровень 1 - отсутствие ходьбы.

\section{Резумьтаты}

Из обследованных 544 пациентов переломы длинных трубчатых костей наблюдались у 134 (204 перелома) детей, которые вошли в исследуемую группу. Ретроспективный анализ историй болезни позволил выделить четыре наиболее часто встречающиеся причины возникновения переломов у детей с последствиями спинномозговой грыжи (рис. 1).

Как видно из рис. 1, наибольшее количество переломов мы наблюдали в процессе и после орто- педического лечения (41\%). При консервативном лечении переломы происходили при этапном гипсовании деформаций стоп, использовании шин, устройств, повязок для стабилизации тазобедренного сустава при наличии подвывиха или вывиха бедра. При хирургическом лечении контрактур и деформаций нижних конечностей, выполнении реконструктивных операций на тазобедренном и коленном суставах, а также стабилизирующих операций на стопах переломы возникали в послеоперационном периоде.

Мы изучили зависимость частоты переломов у детей с последствиями спинномозговой грыжи от различных факторов. У большинства пациентов с переломами различной локализации отмечался грудной и $\mathrm{L}_{1}-\mathrm{L}_{2}$ нейросегментарный уровень - у 49 из 110 (44,5\%) и у 32 из 84 (38,1\%) детей соответственно. В то же время у пациентов с нейросегментарным уровнем $\mathrm{S}_{2}$ переломы наблюдались всего лишь в 3 из 79 (3,8\%) случаев. Таким образом, выявлена прямая закономерность: чем ниже нейросегментарный уровень, тем меньше частота встречаемости перелома (табл. 2).



Рис. 1. Причины формирования переломов 
Распределение пациентов с переломами Алинных трубчатых костей

в зависимости от нейросегментарного уровня

\begin{tabular}{|c|c|c|c|}
\hline $\begin{array}{c}\text { Нейросегментарный } \\
\text { уровень }\end{array}$ & Всего пациентов & $\begin{array}{c}\text { Всего пациентов } \\
\text { с переломами }\end{array}$ & $\%$ \\
\hline Грудной & 110 & 49 & 44,5 \\
\hline $\mathrm{L}_{1}-\mathrm{L}_{2}$ & 84 & 32 & 38,1 \\
\hline $\mathrm{L}_{3}-\mathrm{L}_{4}$ & 186 & 38 & 20,4 \\
\hline $\mathrm{L}_{5}-\mathrm{S}_{1}$ & 85 & 12 & 3,1 \\
\hline $\mathrm{S}_{2}$ & 79 & 3 & 3,8 \\
\hline
\end{tabular}

Таблица 3

Аокализация перелома кости в зависимости от нейросегментарного уровня

\begin{tabular}{|c|c|c|c|}
\hline Нейросегментарный уровень & Перелом бедренной кости & Перелом костей голени & Всего \\
\hline Грудной & $65(79,3 \%)$ & $17(20,7 \%)$ & $82(100 \%)$ \\
\hline $\mathrm{L}_{1}-\mathrm{L}_{2}$ & $42(76,4 \%)$ & $13(23,6 \%)$ & $55(100 \%)$ \\
\hline $\mathrm{L}_{3}-\mathrm{L}_{4}$ & $26(52 \%)$ & $24(48 \%)$ & $50(100 \%)$ \\
\hline $\mathrm{L}_{5}-\mathrm{S}_{1}$ & $2(14,3 \%)$ & $12(85,7 \%)$ & $14(100 \%)$ \\
\hline $\mathrm{S}_{2}$ & - & $3(100 \%)$ & $3(100 \%)$ \\
\hline Всего & $135(66,2 \%)$ & $69(33,8 \%)$ & $204(100 \%)$ \\
\hline
\end{tabular}

Таблица 4

Особенности переломов дминных трубчатых костей у детей с последствиями спинномозговых грыж

\begin{tabular}{|l|c|c|}
\hline \multicolumn{1}{|c|}{ Симптом/признак Клинические особенности } & Атипичный перелом \\
\hline Боль & + & + \\
\hline Посттравматическая деформация & + & - \\
\hline Отек в первые часы после травмы & + & - \\
\hline Адекватность травмы & + & - \\
\hline & + & \\
\hline Линия перелома & + & - \\
\hline Чрезмерная периостальная реакция & - & + \\
\hline Участки склероза в месте перелома & + \\
\hline
\end{tabular}

Мы также проанализировали зависимость частоты локализации травмы нижней конечности (бедро/голень) от нейросегментарного уровня и установили, что переломы бедренной кости чаще наблюдались у пациентов при грудном и $\mathrm{L}_{1}-\mathrm{L}_{2}$ нейросегментарном уровне - в 79,3 и 76,4\% соответственно, тогда как у пациентов $c \mathrm{~L}_{5}-\mathrm{S}_{1}$ нейросегментарным уровнем чаще встречались переломы костей голени - в 85,7 \% (табл. 3).
Таким образом, чем ниже нейросегментарный уровень, тем меньше частота встречаемости перелома бедренной кости. С учетом того что в большинстве случаев нейросегментарный уровень последствий спинномозговой грыжи определяет двигательные возможности ребенка (а они тем выше, чем ниже нейросегментарный уровень), такие особенности топического расположения переломов мы связываем именно с этим фактом. Так, у 15 пациентов с нейросегментарным уровнем 
$\mathrm{L}_{5}-\mathrm{S}_{1}$ и $\mathrm{S}_{2}$ переломы наблюдались при падении во время передвижения.

У 22 (16\%) пациентов имелось 3 и более переломов. У 98 из 134 (73\%) детей двигательные возможности соответствовали 1-му и 2-му уровню двигательной активности. У 48 (36\%) переломы наблюдались после длительного периода гипсовой иммобилизации с целью коррекции контрактур и деформации нижних конечностей. У 22 из 134 (16\%) пациентов в ближайшие 6 месяцев после первичного перелома наблюдался повторный перелом, при этом наиболее часто - перелом бедренной кости в нижней ее трети.

Клиническая картина перелома у ребенка со спинномозговой грыжей имела ряд особенностей. В первую очередь зачастую отсутствовал классический анамнез и клинические признаки повреждения: в $19 \%$ случаев возникали «спонтанные» переломы, под которыми мы подразумевали переломы без наличия достоверного травматического фактора. Патологической подвижности в месте перелома не было в $56 \%$ случаев, отек отсутствовал у $88 \%$ детей, а боль в области перелома мы наблюдали только в 19 \% случаях.

Сравнительные признаки типичного и атипичного течения перелома представлены в табл. 4 .

Отличительной особенностью атипичного перелома у данной категории пациентов также являлось отсутствие адекватной травмы, или факт травмы был не замечен пациентами или родителями. Отсутствие боли в области перелома связано с нарушением чувствительности, вызванным основным заболеванием, отсутствие или незначительная величина посттравматической деформации конечности - с неполным разобщением костных фрагментов перелома ввиду низкой двигательной активности пациентов. Косвенные клинические признаки перелома, а также изменения в общем анализе крови (лейкоцитоз, тромбоцитоз и увеличение СОЭ) должны служить основанием для рентгенологического обследования.

Среди рентгенологических особенностей атипичного перелома длинных трубчатых костей у детей с последствиями спинномозговых грыж мы отметили: отсутствие линии перелома, наличие избыточной периостальной реакции, а также участки склероза в месте перелома (рис. 2).

Гипертрофическая костная мозоль определялась у 31 из 134 (23\%) пациентов. При ретроспективном анализе было установлено, что нетипичный характер рентгенологической картины перелома у 10 пациентов потребовал проведения костной биопсии с целью исключения

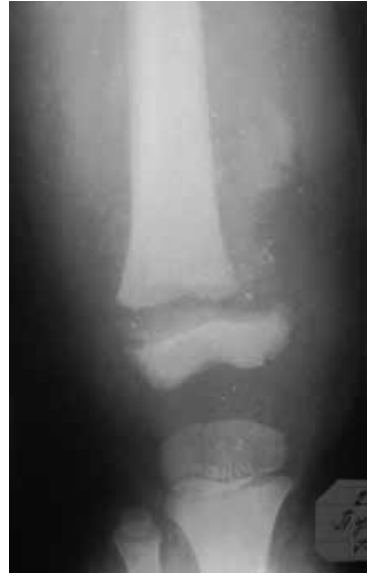

$a$

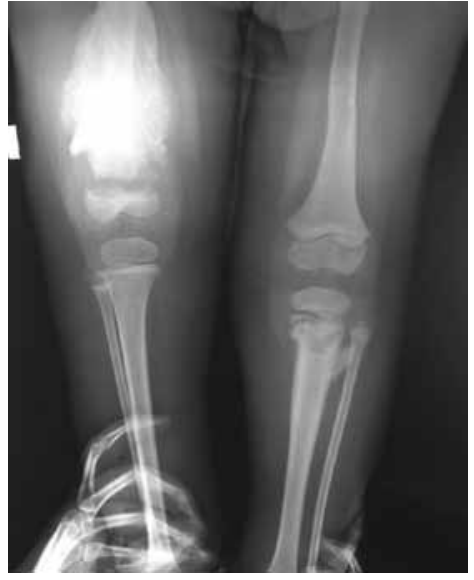

6
Рис. 2. Пациентка Г. Диагноз: «Последствие спинномозговой грыжи. Нейросегментарный уровень $\mathrm{L}_{1}-\mathrm{L}_{2}$. Остеоэпифизеолиз дистального эпифиза бедренной кости справа»: $a-14$-е сутки после травмы; 6 - через 5 недель после травмы, гипертрофическая периостальная реакция

остеогенной саркомы, а у 5 пациентов избыточная костная мозоль была ошибочно трактована как проявление остеомиелита. Знание рентгенологических особенностей атипичных переломов длинных трубчатых костей у пациентов с последствиями спинномозговых грыж позволяет провести правильную дифференциальную диагностику и выработать соответствующую тактику лечения.

\section{ЗакАючение}

У детей с последствиями спинномозговых грыж наблюдаются различные ортопедические проблемы, проявляющиеся в течение всей жизни. Переломы длинных трубчатых костей нижних конечностей у таких пациентов становятся частым осложнением в процессе ортопедического лечения. Данная проблема в отечественной и зарубежной литературе представлена единичными публикациями, затрагивающими частные вопросы диагностики и лечения переломов длинных трубчатых костей. Проведенное нами исследование подтверждает высокую распространенность переломов у пациентов с последствиями спинномозговых грыж. Установлено, что их частота и локализация напрямую зависят от нейросегментарного уровня, который в свою очередь определяет уровень двигательной активности детей, являющийся одним из факторов развития остеопороза. Результаты исследования подчеркивают необходимость соблюдения особой осторожности с целью предупреждения переломов у таких детей на разных этапах лечения. Хирургическое лечение деформаций нижних конечностей необходимо вы- 
полнять с учетом потенциальных двигательных возможностей ребенка, оценивая реабилитационный потенциал пациента с помощью классификации Sharrard.

\section{Выводы}

1. У детей с последствиями спинномозговой грыжи в зависимости от нейросегментарного уровня поражения спинного мозга наблюдается различная степень снижения уровня двигательной активности и осевой нагрузки на костно-суставной аппарат, что является одним из основных факторов риска развития вторичного остеопороза.

2. Частота и локализация переломов длинных трубчатых костей нижних конечностей у детей с последствиями спинномозговой грыжи находятся в прямой зависимости от нейросегментарного уровня.

3. Клиническая картина атипичного перелома нижних конечностей у ребенка с последствием спинномозговой грыжи отличается отсутствием болевого синдрома, отека в области перелома, смещения костных отломков, что необходимо учитывать при диагностике.

4. Рентгенологическая картина перелома у детей с последствиями спинномозговой грыжи характеризуется особенностями образования костной мозоли (отсутствие четкой линии перелома, наличие избыточной периостальной реакции, участки склероза в месте перелома), которые необходимо учитывать в процессе диагностики и лечения.

\section{Аополнительная информация}

Источник финансирования. Настоящая работа выполнена в рамках НИР ФГБУ «НИДОИ им. Г.И. Турнера» Минздрава России.

Конфликт интересов. Авторы декларируют отсутствие явных и потенциальных конфликтов интересов, связанных с публикацией настоящей статьи.

Этическая экспертиза. Все пациенты и их законные представители дали согласие на обработку и публикацию персональных данных.

\section{Вклад авторов.}

А.С. Федосеева, М.А. Угурчиева и Т.Н. Прокопенко - сбор и обработка клинического материала.

B.M. Кенис и С.В. Иванов - написание результатов и выводов представленной статьи.

Благодарности. Коллектив авторов выражает благодарность ученому секретарю ФГБУ «НИДОИ им. Турнера» Минздрава России, канд. мед. наук Алле Владимировне Овечкиной за помощь в оформлении научной работы.

\section{Список митературы}

1. Баиндурашвили А.Г., Иванов С.В., Кенис В.М. Подвывих и вывих бедра у детей с последствиями спинномозговых грыж (Обзор литературы) // Травматология и ортопедия России. 2013. - № 4. - C. 97-102. [Baindurashvili AG, Ivanov SV, Kenis VM. Subluxation and dislocation of the hip in children with spina bifida (Review). Travmatologiia $i$ ortopediia Rossii. 2013;(4):97-102. (In Russ.)]

2. Баиндурашвили А.Г., Иванов С.В., Кенис В.М. Нейросегментарный уровень и его значение при лечении подвывиха и вывиха бедра у детей с последствиями спинномозговых грыж // Ортопедия, травматология и восстановительная хирургия детского возраста. - 2016. - Т. 4. - № 4. - С. 6-11. [Baindurashvili AG, Ivanov SV, Kenis VM. Clinical implications of the neurosegmental level of injury in the treatment of hip dislocation and subluxation in children with spina bifida. Pediatric traumatology, orthopaedics and reconstructive surgery. 2016;4(4):6-11. (In Russ.)]. doi: 10.17816/PTORS446-11.

3. Иванов С.В., Кенис В.М., Икоева Г.А., Коченова Е.А. Использование роботизированной двигательной реабилитации в комплексном лечении подвывиха и вывиха бедра у детей с последствиями спинномозговой грыжи // Ортопедия, травматология и восстановительная хирургия детского возраста. - 2014. - T. 2. - № 4. - С. 32-35. [Ivanov SV, Kenis VM, Ikoeva GA, Kochenova EA. The use of robotic rehabilitation in complex treatment of subluxation and dislocation of the hip in children with sequelae of spina bifida. Pediatric traumatology, orthopaedics and reconstructive surgery. 2014;2(4):32-35. (In Russ.)]. doi: 10.17816/PTORS2432-35.

4. Щеплягина Л.А., Моисеева Т.Ю. Проблемы остеопороза в педиатрии: возможности профилактики // РМЖ. - 2003. - Т. 11. - № 27. - С. 1554-1556. [Scheplyagina LA, Moiseeva TY. Problemy osteoporoza v pediatrii: vozmozhnosti profilaktiki. RMZh. 2003;11(27):1554-1556. (In Russ.)]

5. Yasar E, Adiguzel E, Arslan M, Matthews DJ. Basics of bone metabolism and osteoporosis in common pediatric neuromuscular disabilities. Eur J Paediatr Neurol. 2018;22(1):17-26. doi: 10.1016/j.ejpn.2017.08.001.

6. Пырьева Е.А., Сорвачева Т.Н., Сафронова А.Н. Нутритивная поддержка в лечении детей с неврологической патологией // Вопросы детской диетологии. - 2016. - Т. 14. - № 1. - С. 47-53. [Pyreva EA, Sorvacheva TN, Safronova AN. Nutritive support in treatment of children with neurological pathology. Problems of Pediatric Nutritiology. 2016;14(1):47-53. (In Russ.)]. doi: 10.20953/1727-57842016-1-47-53.

7. Kilpinen-Loisa P, Pihko H, Vesander U, et al. Insufficient energy and nutrient intake in children with mo- 
tor disability. Acta Paediatr. 2009;98(8):1329-1333. doi: 10.1111/j.1651-2227.2009.01340.x.

8. Lock TR, Aronson DD. Fractures in patients who have myelomeningocele. J Bone Joint Surg Am. 1989;71(8):1153-1157.

9. Marreiros H, Loff C, Calado E. Osteoporosis in paediatric patients with spina bifida. J Spinal Cord Med. 2012;35(1):9-21. doi: 10.1179/2045772311Y.0000000042.
10. Hara H, Matsumoto S, Ohishi T, et al. Posterior Iliopsoas Transplantation in the Treatment of Paralytic Hip Dislocation in Spina Bifida. Orthop Traumatol. 1980;29(4):718-721. doi: 10.5035/nishiseisai.29.718.

11. Pauly M, Cremer R. Levels of mobility in children and adolescents with spina bifida-clinical parameters predicting mobility and maintenance of these skills. Eur J Pediatr Surg. 2013;23(2):110-114. doi: 10.1055/ s-0032-1324689.
Станислав Вячеславович Иванов - канд. мед. наук, руководитель Центра Spina bifida отделения № 4 ФГБУ «НИДОИ им. Г.И. Турнера» Минздрава России, Санкт-Петербург. ORCID 0000-0002-2187-3973. E-mail: ortostas@mail.ru.

Владимир Маркович Кенис - д-р мед. наук, доцент, заместитель директора по развитию и внешним связям, руководитель отделения № 4 ФГБУ «НИДОИ им. Г.И. Турнера» Минздрава России, Санкт-Петербург. ORCID: https://orcid.org/0000-0002-7651-8485. E-mail: kenis@mail.ru.

Татьяна Николаевна Прокопенко - врач-педиатр консультативно-диагностического центра ФГБУ «НИДОИ им. Г.И. Турнера» Минздрава России, Санкт-Петербург. E-mail: prokopenkotn@mail.ru.

Александра Сергеевна Федосеева - врач-травматологортопед СПбГБУЗ «Детская городская больница Святой Ольги». ORCID 0000-0001-5196-4748. E-mail: dok72101@ mail.ru.

Милана Ахятовна Угурчиева - ординатор кафедры «Травматологии и ортопедии» ФГБОУ ВО «ПСПбГМУ им. акад. И.П. Павлова» Минздрава России. ORCID 0000-0002-1888-4866. E-mail: m110393@mail.ru.
Stanislav V. Ivanov - MD, PhD, Head of the Spina Bifida Center of the Department No 4. The Turner Scientific Research Institute for Children's Orthopedics, Saint Petersburg, Russia. ORCID 0000-0002-2187-3973. E-mail: ortostas@mail.ru.

Vladimir M. Kenis - MD, PhD, Associate Professor, Deputy Director for Development and International Relations, Head of the Department N 4. The Turner Scientific Research Institute for Children's Orthopedics, Saint Petersburg, Russia. ORCID: https://orcid.org/00000002-7651-8485. E-mail: kenis@mail.ru.

Tatyana N. Prokopenko - MD, Pediatrician of the Consultative and Diagnostic Center of The Turner Scientific Research Institute for Children's Orthopedics, Saint Petersburg, Russia. E-mail: prokopenkotn@mail.ru.

Aleksandra S. Fedoseyeva - MD, Orthopedic and Trauma Surgeon. Children's City Hospital of St. Olga, Saint Petersburg, Russia. ORCID 0000-0001-5196-4748. E-mail: dok72101@mail.ru.

Milana A. Ugurchieva - MD, Resident of the Department of Traumatology and Orthopedicsthe of the I.P. Pavlov. St. Petersburg State Medical University under the Ministry of Health of Russia. ORCID 0000-0002-1888-4866. E-mail: m110393@mail.ru. 